\title{
IS LEP BEAM-BEAM LIMITED AT ITS HIGHEST ENERGY?
}

\author{
D. Brandt, W. Herr*, M. Meddahi and A. Verdier, CERN, Geneva, Switzerland
}

\section{ABSTRACT}

The operation of LEP at $45.6 \mathrm{GeV}$ was limited by beambeam effects and the vertical beam-beam parameter $\xi_{y}$ never exceeded 0.045. At the highest energy of $94.5 \mathrm{GeV}$, the increased damping allows higher beam-beam parameters $\xi_{y}$. Values above 0.07 in the vertical plane averaged over four experiments have been obtained frequently with peak values up to 0.075 in a single experiment. Although the maximum intensity in LEP is presently limited by technical considerations, some observations indicate that the beam-beam limit is close and the question of the maximum possible values can be raised. These observations are shown in this paper and possible consequences are presented. The optimum operation of LEP in the neighbourhood of the beam-beam limit is discussed.

\section{TUNE SHIFT AND BEAM-BEAM LIMITS}

\subsection{Beam-beam tune shift}

For stable motion, the relation between the tune shift $\Delta Q$ and the beam-beam parameter $\xi$ is [1]:

$$
\cos (2 \pi Q+2 \pi \Delta Q)=\cos (2 \pi Q)-2 \pi \xi \sin (2 \pi Q)
$$

and

$$
\beta^{*}=\frac{\beta \sin (2 \pi Q)}{\sin (2 \pi Q+2 \pi \Delta Q)}
$$

where $Q$ is the tune of the machine and $\beta$ and $\beta^{*}$ are the unperturbed and perturbed $\beta$-functions at the collision point. The unperturbed beam-beam parameter $\xi$ is written as:

$$
\xi_{x, y}=\frac{N r_{e} \beta_{x, y}}{2 \pi \gamma \sigma_{x, y}\left(\sigma_{x}+\sigma_{y}\right)}
$$

For small tune shifts, it can be shown that $\Delta Q \approx \xi$, but is different for large values. The perturbed $\beta^{*}$ can be written as [1]:

$$
\beta^{*}=\frac{\beta}{\sqrt{1+4 \pi \xi\left(\cot \left(2 \pi Q^{i}\right)\right)-4 \pi^{2} \xi^{2}}}
$$

where $2 \pi Q^{i}$ is the phase advance between two interaction points. The tune shift is therefore a function of the tune which can be chosen to keep the actual beam-beam tune shift small, i.e. a working point close to the integer is

\footnotetext{
*Email: Werner.Herr@cern.ch
}

preferable unless this is prohibited for other considerations. Since the tune shift is amplitude dependent, it will also create a tune spread which cannot be corrected. While for small $\xi$ the tune shift and $\beta^{*}$ hardly change over a large range of the phase advance, for larger $\xi$ this variation is strong and therefore for the high values of beam-beam parameters now observed at LEP, one can expect significant effects on the optics and beam dynamics. For LEP, the vertical tune is 96.19 and Fig.1 shows the vertical tune shift $\Delta Q_{y}$ as a function of the unperturbed beam-beam parameter $\xi_{y}$ for this tune value. For the maximum beam-beam parameter observed in LEP, i.e. 0.070, the tune shift is around 0.05 and $\beta_{y}^{*}$ is reduced from $5 \mathrm{~cm}$ to $2.8 \mathrm{~cm}$ at the collision points for small amplitude particles. It is this actual tune shift $\Delta Q_{y}$ which is important for the evaluation of resonances excited by the beam-beam force while the beam-beam parameter $\xi$ merely measures the strength of the beam-beam effect and could be used to compare operational performance. The LEP2 values can easily compete

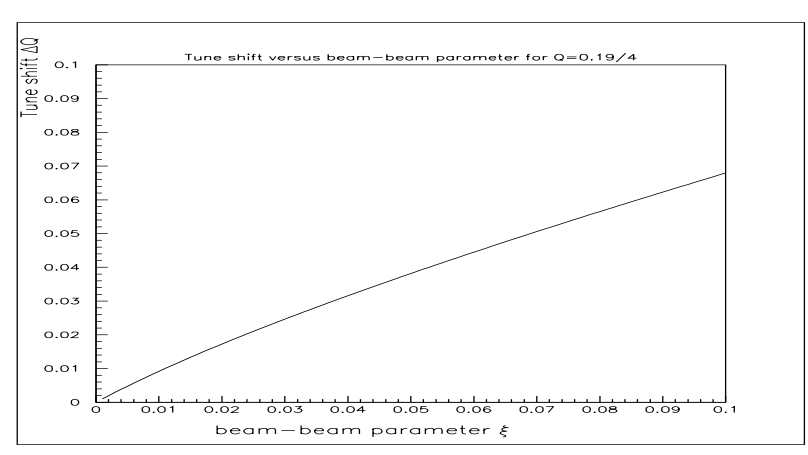

Figure 1: Beam-beam tune shift as function of beam-beam parameter for typical LEP tune

with previously achieved records: at ADONE the beambeam parameter was as high as 0.08 , but the tune shift $\Delta Q_{y}$ was around 0.03 [2], i.e. significantly lower than for LEP2. During a dedicated experiment with round beams [3] at CESR, a beam-beam parameter up to 0.09 was measured, however with rather large $\beta_{y}^{*}$, therefore low luminosity and only a single experiment. The LEP2 values are the highest observed in high luminosity operation.

\section{2 "Strong" beam-beam limit}

What is generally recognized as a signature of the beambeam limit is a linear rather than a quadratic increase of the 
luminosity with intensity. This is interpreted as a saturation of $\xi$ with the bunch intensity and an emittance increase with increasing intensity. It is desirable to operate LEP at or near this "first beam-beam limit" since the luminosity would reduce only linearly with decreasing bunch intensity and give a larger integrated luminosity. Various tools such as e.g. artificial emittance increase with wigglers, damping partition change, coupling and optics are used to control this limit. However, this limit is not very sharp and already well below other effects may be observed. Particles at large amplitudes exhibit an unstable behaviour, leading to the formation of tails and a decreased life time. This can usually be understood by resonances exciting particles at large amplitude. Together with a reduced dynamic aperture this "second beam-beam limit" may be found early and the region of constant $\xi$ cannot be reached. Therefore care must be taken to provide sufficient dynamic aperture when the machine is operated close to the beam-beam limit. In LEP both effects were clearly observed.

\section{3 "Weak" beam-beam limit}

Apart from the classical beam-beam limits discussed above, a strong beam-beam interaction can cause effects on the beam dynamics which manifest themselves in other types of limitations or operational difficulties, eventually leading to a limited performance. Signs of such weak limits are clearly observed in LEP. The coherent beam-beam effect can limit the available space in the working diagram significantly and beam-beam induced orbit distortions lead to collision offsets or parameter splitting between the two beams. Such effects eventually limited the performance of LEP running with bunch trains [4]. The excitation of coherent modes is usually damped with a finite chromaticity, however significant chromaticity splits between the two beams caused by strong beam-beam effects can severely limit this possibility. As it was already mentioned, the beam-beam interaction perturbs the beam optics in a predictable way. Combined with possible imperfections such as e.g. phase errors or collision offsets this can lead to significant distortions and limit the overall performance. This will be treated in more detail in a later section.

\section{EXPERIENCE AT 45.6 GEV}

LEP was run for 7 years at $45.6 \mathrm{GeV}$, corresponding to the $\mathrm{Z}^{0}$ resonance. Since beam-beam effects are stronger at lower energies, they were the main intensity limitation. Details about running LEP at lower energy and the operational procedures can be found in [5] and are not discussed here. Only the main observations are summarized.

Already at rather low intensities LEP showed the typical behaviour of operation at the beam-beam limit, i.e. saturation of the beam-beam parameter $\xi$. Using wigglers to control the horizontal beam size allowed to run at the limit for several hours and the ultimate limit for the bunch intensity was due to the significant amount of non-Gaussian tails. For most of the time an optics with phase advance of $90^{\circ} / 60^{\circ}$ in the horizontal and vertical planes was used which had a sufficient dynamic aperture and the region of constant $\xi$ was reached at bunch intensities around $400 \mu \mathrm{A}$, corresponding to $\xi_{y}^{\max } \approx 0.045$.

\section{OBSERVATIONS AT HIGH ENERGY}

With increasing energy the effects of synchrotron radiation increase rapidly. For a given optics, the horizontal emittance increases with $\mathrm{E}^{2}$ and therefore the horizontal beambeam parameter decreases with $\mathrm{E}^{-3}$. Special low emittance optics have been developped for high energies [7] to compensate for the emittance increase. The damping times also decrease with $\mathrm{E}^{-3}$ and one can expect that larger values for $\xi$ can be obtained before the beam-beam limit is reached because the effects of resonances are suppressed.

\subsection{Luminosity and tune shift}

The vertical beam-beam parameter $\xi_{y}$ is computed from the luminosity measured by the experiments, using the measured bunch intensity, the unperturbed $\beta_{y}$ and the theoretical horizontal emittance. All values quoted are averaged over the 4 experiments and 2 minutes. With this procedure maximum values of 0.07 for $\xi_{y}$ and around 0.05 for $\xi_{x}$ have been obtained at $94.5 \mathrm{GeV}$. The highest value was 0.075 , however only in a single experiment and for less than one minute. The Fig. 2 shows the evolution of the vertical beam-

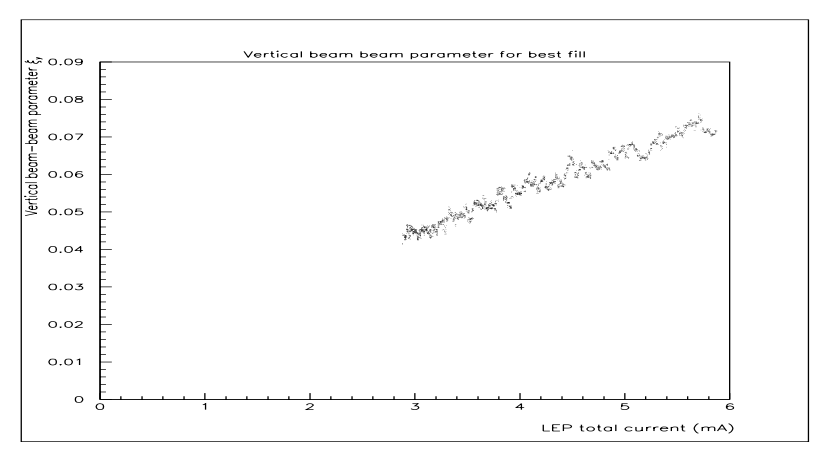

Figure 2: Vertical beam-beam parameter $\xi_{y}$ as function of intensity for one fill

beam parameter during one of the best fills as a function of the decreasing bunch intensity. No sign of saturation can be seen up to the maximum of $\xi_{y}=0.07$ and $\xi$ seems to linearly decrease with the intensity. At this energy LEP is operated with four bunches per beam and the current per bunch at the start of a fill was approximately $750 \mu \mathrm{A}$. From Fig. 1 it can be derived that the tune shift per interaction point is around 0.05 , i.e. a very significant part of the tune space is occupied by the beam-beam tune spread. For both planes it is increasingly difficult to find a working point to avoid resonances. It was demonstrated [6] that in particular the background generated from tails or coherent motion is extremely sensitive to the choice of the horizontal working 
point. This requires a continuous monitoring and adjustment of the tune while it changes with decreasing current.

\subsection{Optics dependence of beam-beam effects}

The effects of the beam-beam interaction depend on the optics used. In particular they depend on the phase advance between the interaction points, the lattice non-linearities and imperfections. In the course of the search for an appropriate low emittance lattice it was found that the beambeam interaction can create or enhance beam tails which can limit the performance of the machine when the dynamic aperture is not sufficiently large. This effect and its dependence on the optics parameters is treated in another paper [7].

\subsection{Optical functions at interaction point}

The beam-beam forces at the interaction points act as nonlinear lenses and therefore distort the optics. The most obvious manifestation of these distortions is the tune shift $\Delta Q$ and the amplitude dependent beating of the optics functions. Such effects are non-negligible and must be taken into account for beam measurements, such as e.g. emittance measurements $[8,9]$. The change of the $\beta$-functions at the interaction points can be calculated as shown above for small amplitude particles: without any further imperfections, the effect of four symmetric beam-beam collisions is a decrease of the horizontal and vertical $\beta$. This effect is equal for all collision points when the phase advance between them is equal (eq. (4)). Phase advance errors between the collision points can break this symmetry. A "phase bump", i.e. a small phase advance error between two interaction points and compensated in the following arc usually introduces a global beating and reduces the $\beta$ symmetrically everywhere. In the presence of beambeam interactions however, this symmetry is broken and $\beta$ is drastically decreased in one single interaction point, possibly leading to different luminosities. Such imbalanced luminosities have been observed during a large part of LEP running in 1998 [10] and beam-beam induced optics distortions remain a possible explanation. A similar effect is

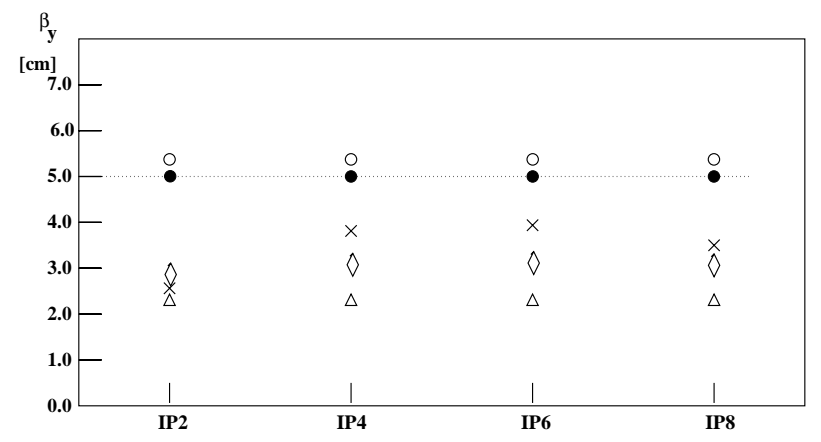

Figure 3: Optical functions at interaction point modified by beam-beam interactions reproduced by small collision offsets. While for head on collisions the modification of the optics function is fully symmetric, with small collision offsets of the order of the beam size a strong beating is introduced, similar to that due to phase advance errors. The correction of a collision offset in one collision point will therefore also improve the performance in the others. These effects are summarized for one example in Fig.3. The value of $\beta_{y}$ is shown for the four interaction points under different conditions. The unperturbed values are indicated as $(\bullet)$ and the effect of a pure phase advance error before and after IP4 as ( $($ ). The phase error introduced to produce this effect was around $10^{\circ}$. Further we show the effect of a symmetric beam-beam interaction without imperfections $(\triangle)$, phase advance error with beam-beam $(\times)$ and beam-beam with a small offset in IP2 $(\diamond)$. It is clear that running LEP in the strong beam-beam regime requires a careful setting of the machine parameters, in particular phase advance errors or collision offsets must be avoided.

\section{CONCLUSION}

The beam-beam effect in LEP was analysed as a function of the bunch intensity and no sign of saturation of the luminosity or the beam-beam parameter was found. Although LEP is therefore not beam-beam limited in the classical sense, the very strong beam-beam effect with beambeam parameters above 0.07 is the origin of other effects which indirectly limit the performance and constitute therefore a "beam-beam induced" limit, or a "weak" beam-beam limit.

\section{REFERENCES}

[1] A. Chao; Beam-beam instability; in Phys. of High Energy Part. Acc., AIP Conf. Proc. 127, (1983).

[2] M. Basetti; Numerical Computation of space charge effects in a positron and electron storage ring; in Proc. 5th Int. Conf. on High Energy Particle Accelerators, Frascati, (1965).

[3] E. Young et al.; Collisions of resonantly coupled round beams at the Cornell electron-positron storage ring (CESR);

[4] B. Goddard et al.; Bunch trains for LEP; Part. Accelerators Vol.57, Number 4, p. 237, (1998). in Proc. PAC 1997, Vancouver 1997, (1997).

[5] H. Burkhardt; Energy dependence of beam-beam interactions in LEP; in Proc. PAC 1997, Vancouver 1997, (1997).

[6] G. v.Holtey; Protection of LEP experiments against particle backgrounds at highest beam energies; these proceedings.

[7] D. Brandt et al.; Experience with a low emittance optics in $L E P$; these proceedings.

[8] E. Keil; Optics imperfections; Proc. 3th workshop on LEP performance, Chamonix 1993.

[9] P. Castro; How does $\beta$-beating affect the luminosity ?; Proc. 4th workshop on LEP performance, Chamonix 1994.

[10] G. Morpurgo; Do we understand the luminosities imbalance in LEP; Proc. 9th workshop on LEP performance, Chamonix 1999. 